\title{
Problemas na aprendizagem de matemática: uma proposta de ensino usando videoaulas
}

\section{Problems in learning mathematics:a teaching proposalusing video lessons}

\author{
Ive Faria \\ Colégio Estadual República de Cabo Verde \\ ivepina@gmail.com \\ orcid.org/0000-0002-1657-7811
}

\section{Cláudia Concordido}

Instituto de Matemática e Estatística, Universidade do Estado do Rio de Janeiro concordido@ime.uerj.br

\section{orcid.org/0000-0002-0767-9170}

\section{Jeanne Barros}

Instituto de Matemática e Estatística, Universidade do Estado do Rio de Janeiro, Projeto Fundão do Instituto de Matemática, Universidade Federal do Rio de Janeiro jeanne@ime.uerj.br orcid.org/0000-0002-1857-8658

Resumo. O objetivo deste artigo é divulgar uma atividade de recuperação paralela de estudos inovadora que utiliza videoaulas, tema de dissertação de mestrado profissional de Ive Faria em 2017. Foi aplicada para os alunos do $3^{\circ}$ ano do Ensino Médio do Colégio Estadual República de Cabo Verde, de forma semipresencial, sobre o conteúdo de Análise Combinatória, e teve a plataforma YouTube como a principal ferramenta de disseminação do conteúdo. Essa experiência obteve êxito quando foram observados bons resultados em reavaliações com os alunos, mostrando, assim,o potencial devideoaula como instrumento pedagógico no processo de recuperação paralela de estudos.

Palavras-chave: Videoaulas. Recuperação paralela de estudos. Lacunas de aprendizagem. 
Abstract.The aim of this article is to publicize an innovative parallel study recovery activity that uses video lessons, the theme of a professional master's thesis by Ive Faria in 2017. It was applied to the students of the 3rd year of high school at the Colégio Estadual República de Cabo Verde, from semi-face-to-face, on the Combinatory Analysis content, and had the YouTube platform as the main tool for disseminating the content. This experience was successful when good results were observed in reevaluations with students, thus showing the potential of video lessons as a pedagogical tool in the process of parallel retrieval of studies.

Keywords: Video classes. Parallel retrieval of studies. Learning gaps.

Recebido: 01/10/2017 Aceito: 27/10/2017 Publicado: 06/11/2017

\section{Introdução}

Uma frase como "mas eu só errei o sinal" é um tanto quanto comum de se ouvir em uma aula de matemática. Muitas vezes o estudante não tem a menor ideia de quão importante é dominar as regras de sinais para a aprendizagem matemática em qualquer nível de ensino. Isto é, o não entendimento das regras de sinais quando é apresentado o conjunto de números inteiros causa prejuízo na assimilação de qualquer conteúdo que use as regras de sinais, como números reais, equações do primeiro grau, equações do segundo grau, funções, dentre outros. E, por isso, a falta de domínio dessas regras pode levar os alunos a ter um baixo rendimento escolar na série corrente, e também nas séries seguintes, quiçá por toda sua vida acadêmica.

Assim sendo, quando um aluno que está aprendendo sobre o conjunto dos números inteiros tem dificuldade na assimilação das regras de sinais, ele apresenta uma dificuldade normal, decorrente de um desequilíbrio dos processos de desenvolvimento de assimilação e acomodação, definidos por Piaget (ABREU [et al.], 2010, p. 363). Entretanto, se esse mesmo aluno está aprendendo sobre o conjunto dos números racionais e necessita usar as regras de sinais, ensinadas nas aulas do conjunto de números inteiros, mas ele ainda tem dificuldades de utilizá-las, essas dificuldades podem ser classificadas como lacunas de aprendizagem. A expressão lacuna de aprendizagem surgiu da Teoria da Aprendizagem Significativa de Ausubel e decorre de "condiçõesnão atendidas para ocorrência da aprendizagem por compreensão ou por reflexão" (MASINI, 2011, p. 22).

Em matemática, a existência de várias lacunas da aprendizagem, em especial quando já se está no Ensino Médio (EM), é comum, devido aos conceitos matemáticos serem encadeados e interdependentes. De acordo com os Parâmetros Curriculares Nacionais do Ensino Médio (PCNEM):

(...) a Matemática no Ensino Médionão possui apenas o caráter formativo ou instrumental, mas também deve ser vista como ciência, com suas 
características estruturais específicas. É importante que o aluno perceba que as definições, demonstrações e encadeamentos conceituais e lógicos têm a função de construir novos conceitos e estruturas a partir de outros e que servem para validar intuiçoóes e dar sentido àstécnicasaplicadas. (BRASIL, 2000, p. 40-41)

Assim, quando um aluno foi aprovado pelo Conselho Escolar e ficou reprovado em matemática, ou quando um aluno foi aprovado em todas as disciplinas, mas ficou em dependência em matemática, é provável que muitas lacunas da aprendizagem, dentre outros fatores, estejam bloqueando a aprendizagem desse estudante. Mas, se na série seguinte esses conteúdos anteriores não forem recuperados devidamente, a situação se agravará, e a cada nova lacuna, a cada conceito não aprendido, um verdadeiro hiato na aprendizagem matemática desse estudante pode ser criado.

Em geral, as lacunas de aprendizagem são problemas decorrentes de fatores extrínsecos. Portanto, a priori, podem ser pensadas como uma dificuldade escolar (DE). Afinal, a DE tem origem extrínseca, ou seja, relaciona-se com fatores ambientais somados a fatores internos. Esses fatores podem ter origens pedagógicas, socioculturais, econômicas, familiares e/ou emocionais (CARVALHO [et al.], 2007, p. 231). O que nos leva a crer que a $\mathrm{DE}$ pode ser corrigida pedagogicamente. Isso não significa que outras dificuldades escolares não vão ocorrer em algum outro momento, contudo,exatamente aquele problema de aprendizagem pontual terá sido resolvido.

O Colégio Estadual República de Cabo Verde é um colégio de EM, de formação geral, localizado em Irajá, no Rio de Janeiro, por isso, segue a Portaria da Secretaria de Estado de Educação do Rio de Janeiro/ Subsecretaria de Gestão de Ensino (SEEDUC/SUGEN) $n^{\circ} 419 / 2013$. Essa portaria descreve que as ações para a recuperação paralela de estudos devem ser realizadas mediante um Plano Especial de Estudos(PEE) a ser disposto nos regimentos da escola. Isto é, as ações de recuperação de estudos são subsidiadas através de material didático específico construído para esse fim, formando um conjunto de atividades pedagógicas diversificadas (RIO DE JANEIRO, 2013, art.8).

Diante disso, um conjunto de atividades foi criado com o objetivo de atender aos alunos que possuem problemas de aprendizagem(FARIA, 2017). Essas atividades foram aplicadas mediante a criação de um PEE para a recuperação paralela de estudos do $1^{\circ}$ bimestre de 2017, para os alunos do $3^{\circ}$ ano do EM do Colégio Estadual República de Cabo Verde. Esses alunos obtiveram grau abaixo da média estabelecida no Projeto Político Pedagógico (PPP) da escola, ou seja, obtiveram pontuação inferior a cinco pontos. Uma das atividades criadas consistia em assistir avideoaulas do conteúdo de Análise Combinatória, preparadas com base nos Parâmetros Curriculares Nacionais do Ensino Médio (PCNEM) e em técnicas que dariam suporte a quem possuía problemas de aprendizagem. Para que qualquer professor possa reproduzir o processo, foram usados recursos tecnológicos simples e de fácil acesso tanto na gravação quanto na edição das videoaulas. O conjunto de técnicas de cunho teórico e os recursos tecnológicos de cunho técnico utilizados são apresentados para fundamentar as atividades de recuperação paralela de estudos. 


\section{Teoria e prática}

As atividades criadas para a recuperação paralela foram em um total de 4 (quatro), a saber, assistir videoaulas, resolver tarefas presentes nas videoaulas, responder questionário para cada videoaula assistida e a reavaliação. Somente as duas últimas foram obrigatoriamente presenciais. As tarefas resolvidas e as respostas dos questionários são instrumentos de avaliação do tipo portfólio, enquanto a reavaliação é uma prova discursiva do tipo operatória e com consulta direcionada.

\subsection{Videoaulas}

O principal material didático construído para auxiliar as ações de recuperação paralela de estudos desse conteúdo para a turma se constitui de 5 (cinco) videoaulas de Análise Combinatória. A construção teórica das aulas de Análise Combinatória baseou-se no papel da matemática para o EM descrita nos PCNEM objetivando resolver os problemas de aprendizagem dos alunos. Para exemplificar essa construção, a Figura 1 mostra um slide de uma das videoaulas. Nessa, que foi a primeira aula, é explicado como se processa a Análise Combinatória, através de suas várias etapas: o que é, quais são suas possíveis representações, estratégias de resolução dos problemas combinatórios, as relações combinatórias que podem estar envolvidas e os métodos de contagem que auxiliam a resolução desses problemas.

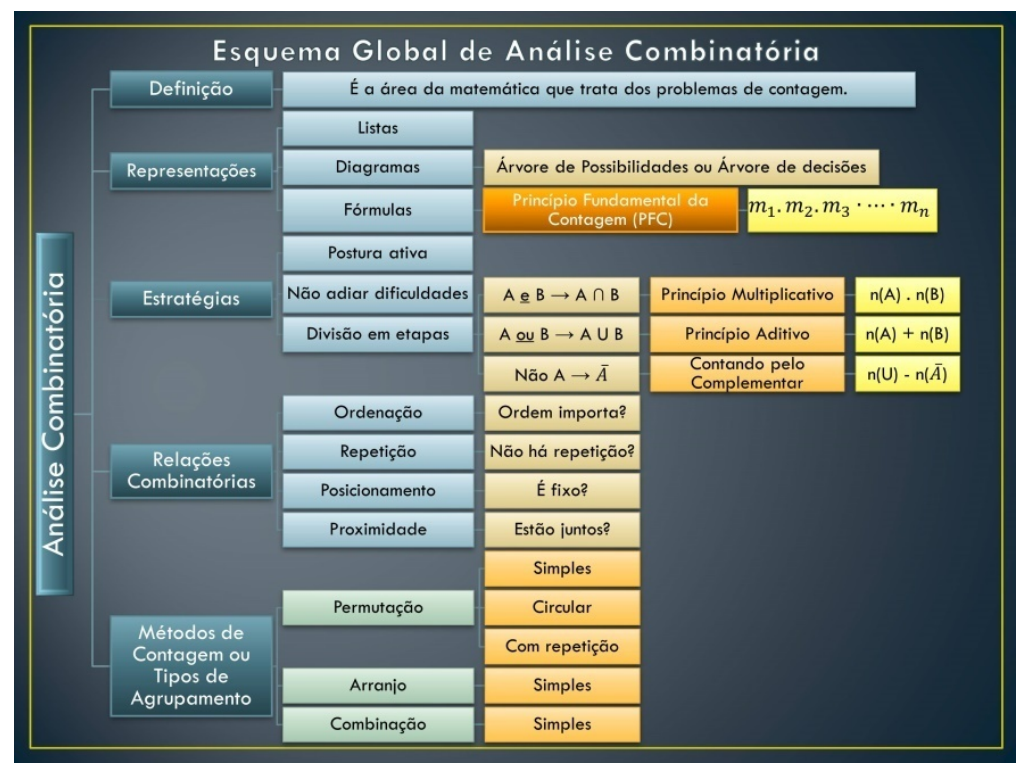

Figura 1.Esquema global de Análise Combinatória.

Fonte: FARIA, 2017.

A Figura 2 traz um exemplo do esquema local de conteúdo de Análise Combinatória que foi tirado da terceira videoaula sobre o conteúdo. A função do esquema local é fazer a ligação das partes com o todo e do todo com as partes do conteúdo. 


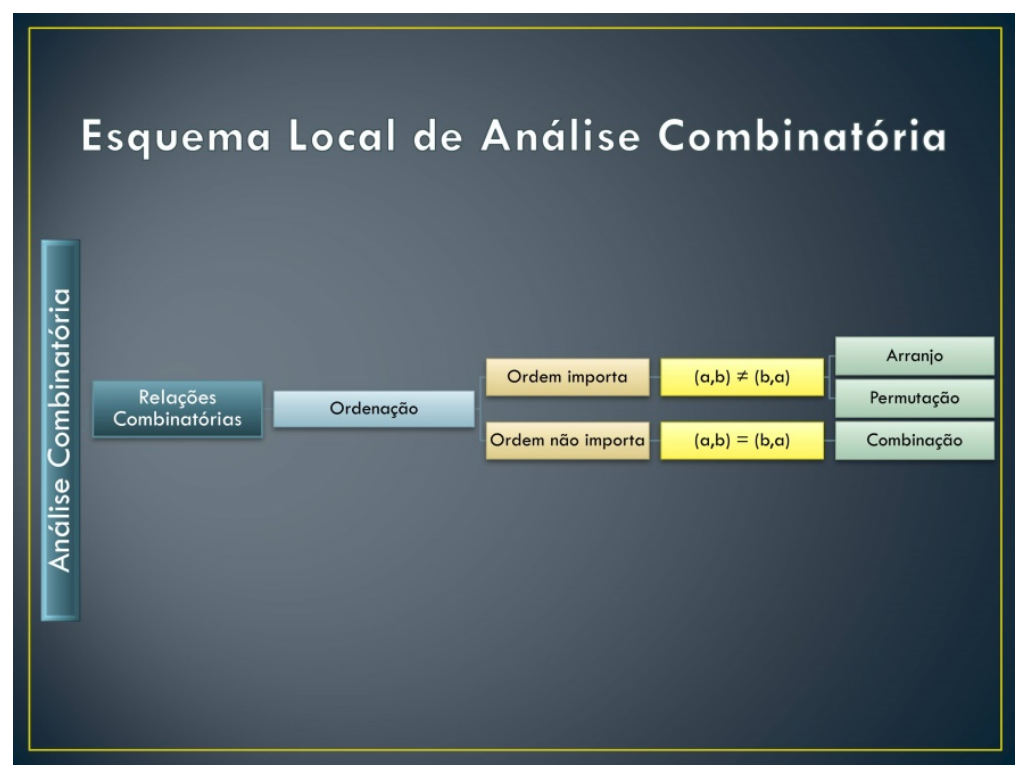

Figura 2. Esquema local de Análise Combinatória.

Fonte: FARIA, 2017.

Os recursos tecnológicos que viabilizaram a gravação e edição das videoaulas foram:

- 1 computador de mesa da marca Positivo, com processador i5, 6 GB de memória, 1 TB de HD, Windows 10, 64 bits.

- 1 notebook da marca LENOVO, com processador i3, 4 GB de memória, 1 TB de HD, Windows 10, 64 bits.

- 1 netbook da marca LG, com processador Intel Atom CPU N270, 2 GB de memória, Windows 7, 32 bits.

- 1 microfone de lapela estéreo, que foi substituído por um microfone de mesa da marca Bright.

- software livre aTube Catcher(captura de vídeo).

- software livre Windows Movie Maker (editor de vídeo da Microsoft).

- software livre Audacity(tratamento de áudio).

Todas as videoaulas estão disponibilizadas na plataforma YouTube $e^{l}$ no canal "Aprenda a estudar matemática". E a divulgação ocorreu mediante link que foi enviado para os alunos pelo WhastApp e, também, ficou disponível na página "Aprenda a estudar matemática" do Facebook ${ }^{2}$, além de estar no Blog ${ }^{3}$ de mesmo nome.A tecnologia da educação foi abordada nesta pesquisa conforme descrevem os autores Ribeiro et al. (2016), Kenski (2005) e Moran (1997), e a ferramenta YouTube foi explorada de acordo com Mattar (2009) e Schmitt (2015).

\footnotetext{
'Disponível em: <https://www.youtube.com/channel/UCv1LAkhGMbbXs2UxXp69WeQ>.

${ }^{2}$ Disponível em: $<$ https://www.facebook.com/aprendaaestudarmatematica $>$.

${ }^{3}$ Disponível em: <http://aprendaaestudarmatematica.blogspot.com.br/>.
} 
Além desse material, foram criados questionários objetivos com a finalidade de verificar se os alunos estavam assistindo de fato às videoaulas. E, em cada videoaula, há questões para serem realizadas como tarefas. Essas ações (de assistir às videoaulas, resolver as tarefas presentes nas videoaulas, responder aos questionários e fazer uma reavaliação) foram pontuadas e constituem o conjunto de atividades pedagógicas diversificadas do PEE que serviu como proposta para integrar o PPP do C. E. República de Cabo Verde (FARIA, 2017, p. 33) naquele dado ano.

A reavaliação, uma avaliação discursiva semelhante a que foi aplicada no primeiro bimestre, serviu como principal instrumento de (re)avaliação de desempenho para todos os alunos que ficaram em recuperação. O objetivo de mantê-la foi verificar a recuperação de conteúdo efetiva dos alunos nesse tipo de avaliação. Porém, nem todos os alunos foram obrigados a participar dessas atividades de recuperação de estudos usando as videoaulas. Alguns optaram por fazer a recuperação paralela de estudos da forma tradicional, ou seja, presencial e através de revisão do conteúdo e reavaliação, conforme previsto no PPP atual da instituição de ensino.Por essa razão, nove alunos (quase $50 \%$ dos alunos em recuperação paralela) assistiram às videoaulas para rever o conteúdo de Análise Combinatória e participaram das atividades propostas (Grupo A) enquanto dez alunos optaram pela forma tradicional de recuperação de estudos (Grupo B). Dessa forma foi possível fazer uma comparação dos resultados desses dois grupos (veja Quadro1).

Quadro 1. Resultados dos alunos antes e depois da avaliação de recuperação.

\begin{tabular}{|c|c|c|c|c|c|c|}
\hline \multicolumn{7}{|c|}{ NOTAS FINAIS DO 10 BIMESTRE } \\
\hline & \multicolumn{3}{|c|}{ GRUPO A } & \multicolumn{3}{c|}{ GRUPO B } \\
\cline { 2 - 7 } & Antes & Depois & \% de melhoria & Antes & Depois & \% de melhoria \\
\hline Aluno 1 & 3,5 & 8,0 & $128,6 \%$ & 3,5 & 6,5 & $85,7 \%$ \\
\hline Aluno 2 & 2,0 & 7,0 & $250 \%$ & 2,0 & 4,0 & $100 \%$ \\
\hline Aluno 3 & 3,5 & 7,0 & $100 \%$ & 2,5 & 4,0 & $60 \%$ \\
\hline Aluno 4 & 4,0 & 6,5 & $62,5 \%$ & 2,0 & 3,5 & $75 \%$ \\
\hline Aluno 5 & 3,5 & 5,5 & $57,1 \%$ & 4,0 & 3,5 & $-12,5 \%$ \\
\hline Aluno 6 & 4,0 & 5,5 & $37,5 \%$ & 2,0 & 2,0 & $0 \%$ \\
\hline Aluno 7 & 0,5 & 4,5 & $800 \%$ & 1,5 & 1,5 & $0 \%$ \\
\hline Aluno 8 & 2,5 & 4,0 & $60 \%$ & 3,5 & 1,5 & $-57,1 \%$ \\
\hline Aluno 9 & 2,0 & 3,0 & $50 \%$ & 3,0 & 1,0 & $-66,7 \%$ \\
\hline Aluno 10 & & & & 4,0 & 1,0 & $-75 \%$ \\
\hline
\end{tabular}

Fonte: FARIA, 2017.

Todos do Grupo A recuperaram suas notas, um deles obteve melhoria de $800 \%$ da nota (antes da prática de recuperação a nota era 0,5 e passou a ser 4,5 , após o processo de recuperação de estudos usando as videoaulas). Enquanto que, no Grupo B, apenas quatro alunos evoluíram, alguns tiveram uma queda de nota que chegou a $66,7 \%$ abaixo da nota antes da recuperação de estudos (FARIA, 2017, p. 61). 


\section{Conclusões}

Os resultados obtidos com a aplicação das atividades mostram que as videoaulas foram eficientes instrumentos de recuperação paralela para os alunos do $3^{\circ}$ ano do EM, pois os alunos que realizaram todas as atividades propostas obtiveram melhoria do desempenho acadêmico se comparados com o outro grupo de alunos (Grupo B). Isso foi comprovado pela recuperação de suas notas, através do somatório da pontuação atribuída às tarefas presentes nas videoaulas, aos questionários com perguntas sobre o que assistiram nas videoaulas e à reavaliação discursiva aplicada.

As videoaulas expositivas de conteúdo de Análise Combinatória foram criadas e disponibilizadas em diversas mídias digitais (YouTube, WhatsApp, Facebook e Blog específico). Afinal, com o surgimento da internet, a capacidade de processamento, armazenamento e transmissão de informações foi revolucionada (RIBEIRO[et al.], 2016, p.1). Apesar de as escolas tradicionais ainda utilizarem muito pouco essas tecnologias para auxiliar a aprendizagem dos alunos, a"tecnologia de informação pode ser utilizada para compensar aquilo que os sistemas convencionais não têm condições de oferecer" (CASTRO (2001) apud RIBEIRO[et al.], 2016, p.1).

As mídias digitais permitem a ampliação da integração da educação presencial e da educação a distância, reunindo o potencial das tecnologias interativas com o melhor da educação tradicional (TORI, 2004, apud KENSKI, 2005, p. 6). E, ainda, se forem usadas continuamente podem possibilitar a formação de cursos híbridos, isto é, o uso das mídias auxiliaria o professor na administração do tempo usado em sala de aula com aulas expositivas e com aulas de verificação do conteúdo de forma dinâmica, através de jogos, oficinas, softwares, dentre outras possibilidades educacionais.

Além disso, o uso das mídias digitais possibilita que alunos e professores realizem suas tarefas em menor tempo e com maior qualidade (RIBEIRO [et al.], 2016, p.3). Ao mesmo tempo, as mídias otimizam o processo de ensino-aprendizagem porque possibilitam a flexibilização do tempo que os alunos destinam para seu estudo (MORAN, 1997, p.1). Cada indivíduo escolherá a hora, equipamento e local que vai usar para executar determinada tarefa educacional. De acordo com Moran (1997, p.2) "a Internet, ao tornar-se mais e mais hipermídia, começa a ser um meio privilegiado de comunicação de professores e alunos".Assim, para que a interação das aulas através das mídias com as aulas presenciais seja um sucesso, o professor deve ter papel de coordenador do processo (MORAN, 1997, p. 4), motivando sempre os alunos.

\section{Financiamento}

O presente trabalho foi realizado com apoio da Coordenação de Aperfeiçoamento de Pessoal de Nível Superior - Brasil (CAPES) - Código de Financiamento 001. 


\section{Referências}

ABREU,L. C. [et al.] A epistemologia genética de Piaget e o construtivismo. Rev. bras. crescimento desenvolvimento humano, São Paulo, v. 20, n. 2, p. 361-366, ago. 2010. Disponível em:

http://pepsic.bvsalud.org/scielo.php?script=sci_arttext\&pid=S010412822010000200018\&lng=pt\&nrm=iso. Acesso em: 13 jul. 2017.

BRASIL. Ministério da Educação. Secretaria de Educação Média e Tecnológica. Parâmetros curriculares nacionais do Ensino Médio. Parte III: Ciências da Natureza, Matemática e suas Tecnologias, p. 40 - 46. Brasília, DF: MEC, 2000. Disponível em: http://portal.mec.gov.br/seb/arquivos/pdf/ciencian.pdf. Acesso em: 11 jul. 2017.

CARVAlHO, F. B. de; CRENITTE, P. A. P.; CiASCA, S. M. Distúrbios de aprendizagem na visão do professor. Revista psicopedagogia, São Paulo, v. 24, n. 75, p. 229-239, 2007.

FARIA, I. S. de P. Problemas na aprendizagem de matemática: uma proposta de ensino usando videoaulas. 2017. 93f. Dissertação (PROFMAT/IME/UERJ), Rio de Janeiro: 2017.

KENSKI, V.M.Gestão e uso das mídias em projetos de educação a distância.Revista ECurriculum,São Paulo, v. 1, n. 1. 2005-2006.

MASINI, E. F. S.Aprendizagem significativa: condições para ocorrência e lacunas que levam a comprometimentos. Universidade Presbiteriana Mackenzie. Aprendizagem Significativa em Revista, São Paulo, v.1. p. 16 - 24, 2011.

MATTAR, J. YouTube na educação: o uso de vídeos em EAD. São Paulo: 2009. Disponível em: $<$ http://www.joaomattar.com/YouTube\%20na\%20Educa\%C3\%A7\%C3\%A3o\%20o\%2 0uso \%20de\%20v\%C3\%ADdeos\%20em\%20EaD.pdf >. Acesso em: 28 jul. 2017

MORAN, J. M. Como utilizar a Internet na educação, Brasília, v. 26, n. 2, 1997. Disponível em: $\quad<$ http://www.scielo.br/scielo.php?script=sci_arttext\&pid=S010019651997000200006\&lng=en\&nrm=iso $>$. Acesso em: 16 jul. 2017.

RIBEIRO, A. G.; ALTINO FILHO, H. V.; ALVES, L. M. N. A tecnologia como instrumento facilitador da relação ensino aprendizagem de matemática. II Seminário Científico da FACIG. Jornada de Iniciação Científica da FACIG. Minas Gerais: 2016.

RIO DE JANEIRO. Secretaria Estadual de Educação. Avaliação interna da aprendizagem: Manual de orientações para operacionalização da Portaria 
SEEDUC/SUGEN No 419/2013. Rio de Janeiro, RJ: SEEDUC, 2013. Disponível em: https://pt.slideshare.net/jsalg/manual-de-operacaionalizao-da-portaria-seeduc-sugen419-2013manual-avaliacao. Acesso em: 08 out. 2017.

SCHMITT, C. M. O YouTube como uma ferramenta pedagógica no ensino de Geografia. Porto Alegre: 2015. Disponível em: https://www.lume.ufrgs.br/bitstream/handle/10183/133982/000978838.pdf?sequence=1. Acesso em: 28 jul. 2017. 